\section{Country life}

A MAGNIFICENT west-facing victorian country residence with two spacious outbuildings in charming mature woodland, conveniently adjoining a golf course on the English South Downs, was demolished recently to make way for a new road. With 60 rooms, more than a kilometre of corridors and no fewer than 225 doors, the entire property was subterranean and owned by a family of badgers (Meles meles). T. J. Roper et al. took advantage of the need to check that the badgers had vacated the premises before the road-building and excavated the sett complex, which consisted of one main sett and two auxiliaries. Such opportunities, though, are rare, and there is little idea of the typical size of a badger sett or even an explanation for the do-it-yourself compulsion that drives the inheritors of these already huge domains to extend and improve them further. Full particulars are on view in J. Zool., Lond. 225, 115-124 (1991).

\section{Feel the length}

How do the filaments in the sarcomere of a striated muscle know when to stop growing? The answer seems to be that the muscle contains measuring rods in the form of extended proteins of enormous length, titin regulating the thick and nebulin the thin filaments. M. Kruger et al. (J. Cell Biol. 115, 97-107; 1991) now come up with the best evidence for this suggestion, using a set of monoclonal antibodies to mark the positions of nebulin epitopes along the sarcomere. The epitopes all remain at a fixed distance from the Z-line (in which the thin filaments are rooted) even when the muscle contracts. Nebulin in situ is at least $0.9 \mu \mathrm{m}$ long; it is clearly an archetype for a molecular ruler.

\section{Neutrino nudge}

THE effects of the $Z^{0}$ particle, the quarry of CERN's LEP collider, have revealed themselves in a nuclear physics experiment at Rutherford-Appleton Laboratory (B. Bodmann et al. Phys Lett. B267, 321-324; 1991). The first evidence of the $Z^{0}$, long before it was actually identified, was the observation in 1972 that neutrinos can bounce off electrons even though they have no electric charge. Until then, neutrinos had been seen only in collisions in which they were consumed (or conversely, created), for example in the neutrinoinduced reverse $\beta$-decay in which chlorine is converted into argon. In the new experiment, a low-energy neutrino passes through a carbon nucleus, but on its way gently nudged the nucleus into an excited state through the 'weak neutral current' mediated by the $Z^{\circ}$. The authors note with satisfaction that the standard model calculations for the interaction's strength work as well at low energy as they do at high-energy.

\title{
Antihydrogen in a new light
}

\section{Richard Hughes}

THE concept of antimatter, introduced by Dirac in 1928, has become so familiar that the use of antiprotons and positrons in high-energy physics experiments is regarded as quite routine. Atomic antimatter, however, has yet to be studied experimentally, largely because of the difficulties involved in producing and manipulating it. But two experimental groups have now reported results ${ }^{1,2}$ that could be applied to the formation of the simplest anti-atom, antihydrogen. Because the spectroscopic techniques that have been developed for hydrogen could be used, this bound state of a positron and an antiproton is the most suitable anti-atom for studying the matterantimatter and other fundamental symmetries of nature.

The formation of antihydrogen by recombination of a positron with an antiproton has an exact analogue in normal matter: the interaction of an electron and a proton to form hydrogen. This process, which has been studied since the earliest years of quantum mech$\operatorname{anics}^{3}$, requires the participation of a third particle for energy and momentum to be conserved. The simplest reaction is radiative recombination, in which a photon is emitted in conjunction with the formation of the atom. But because photon emission from an atom is a relatively slow process, the probability of this reaction is very small unless the velocities of the participating particles are closely matched. For instance, at CERN's Low Energy Antiproton Ring (LEAR), where 5-MeV antiprotons could be combined with a co-moving, intense positron beam, the production rate would be only a few antihydrogen atoms per second.

In 1983, it was suggested that the reaction rate could be enhanced by a factor of about 100 if the interaction region was illuminated with laser photons with energies equal to those of the photons emitted in the radiative recombination ${ }^{4}$. It is this laser-induced or stimulated recombination that has now been demonstrated with electrons and protons. Schramm et al. ${ }^{1}$ have observed laser-induced recombination of $21-\mathrm{MeV}$ protons in a storage ring with co-moving 11.4-keV electrons, to form hydrogen atoms in the state with principal quantum number $n=2$; and Yousif et $a l^{2}$ have demonstrated stimulated recombination of protons from a $400-\mathrm{keV}$ Van der Graaf accelerator with a merged beam of electrons, producing hydrogen in the $n=11$ and 12 levels.

When antihydrogen is eventually produced, the primary focus of experiments with it is likely to be on precision tests of fundamental symmetries. The experimental techniques that have been developed for the optical spectroscopy of hydrogen could be adapted to this purpose, so remarkably high precisions can be anticipated. For instance, the energydifference between the ground state and the metastable $2 s$ level in hydrogen has been measured with a precision of 5 parts in $10^{10}$. Some idea of the precision that is potentially attainable in measurements of this interval comes from noting that the approximately $1 / 8$-second lifetime of the $2 s$ level translates into an intrinsic quantum-mechanical uncertainty of about 1 part in $10^{15}$.

Further improvements in the precision of the hydrogen measurements towards this level will come with developments in optical frequency standards, laser stability and sources of cold atoms. The precision with which the symmetry between matter and antimatter, known as CPT (charge-parity-time), is tested could be improved by several orders of magnitude if such measurements could also be carried out on antihydrogen. This symmetry, which is a consequence of quantum mechanics and relativity, requires that the mass of a particle and its antiparticle be equal, and that their charges be equal and opposite. At present, the masses (really the charge-to-mass ratios) of the proton and antiproton have been shown to be equal to 4 parts in $10^{8}$, from measurements of their cyclotron frequencies in a magnetic field ${ }^{5}$.

Another fundamental principle of physics that could be studied with antihydrogen is whether antiparticles obey the equivalence principle. This principle, which is the foundation of general relativity, requires that all particles in the same gravitational field fall with equal gravitational acceleration. Although there have been many very precise tests of this principle with matter, there has yet to be an experiment with antiparticles such as the positron or antiproton. A measurement of the gravitational acceleration of antihydrogen would offer the advantage of dealing with an electrically neutral particle, so that the influence of stray electric fields would be reduced. Another way to test this principle would be to look at the gravitational shift of precisely measured transition frequencies in hydrogen and antihydrogen. The equivalence principle requires that the shifts be equal in the two systems, and so a test of equivalence for the positron could be deduced from the results.

The recently demonstrated laser- 